\title{
Digital Photo Sharing and Emotions In A Ubiquitous Smart Home
}

\author{
Gaurav Sondhi and Andy Sloane \\ School of Computing and Information Technology, \\ University of Wolverhampton, Wolverhampton, UK
}

\begin{abstract}
Ubiquitous computing has come a long way since Weiser introduced the concept in 1988 where he put forward some ideas as to how the future of computing would allow us to interact more freely. Today this paradigm of computing is affecting us in more than one way from homes, to offices, to travel, to even the way we are presenting ourselves, with smart clothes embedded with technology. When it comes to the home environment we look at objects as personal attributes and form a close relationship with them. Today the $\mathrm{PC}$ has become a personal communication tool, which is being used for variety of purposes like Internet browsing, shopping, photo sharing. The combination of Internet and digital photography has allowed people to share photos with friends and families who are geographically separated. And when we look at smart homes of the future we expect networking and the Internet to form the central hub for information for the homeowners, hence providing an environment for anytime anywhere computing. We aim to provide a ubiquitous photo sharing environment for the smart home of the future which will allow the users to receive, store, and distribute images between various devices and allow them to attach emotions and personal feelings to pictures in a convenient and enjoyable way, as home is a place where we express most of our emotions. This paper provides the design scenario of how the photo-sharing environment will be built and evaluates how social relationships can be enhanced by the use of digital data related to emotions and photographs. We will look at the factors, which govern the use of digital image technology to provide the level of social interaction and entertainment expected within the smart home to support positive ways of living. We believe that a photo-sharing environment for a smart home could help people in providing important reminders and alerts during critical family events.
\end{abstract}




\section{Introduction}

Ubiquitous Computing is concerned with allowing the users timely and efficient access to information that is around us in our daily lives. One of the ways in which this can be achieved is by embedding the information systems into the fabric of our home environment. The technology has to fuse in with the decor of the home so that it can provide constant access to information while being hidden from the user for most of the time. A truly ubiquitous technology is one, which allows the user to ignore its presence, and the user only becomes aware of the system when they interact with it. Information and Communication Technology (ICT) based artifacts and computers would fade into the background while people would be surrounded by intelligent and intuitive interfaces embedded in all kinds of objects [1].

The advent of the Internet has brought this idea of anytime/anywhere access to information a step close within the smart home environment. A "smart home" can be defined as a residence equipped with computing and information technology, which anticipates and responds to the needs of the occupants, working to promote their comfort, convenience, security and entertainment through the management of technology within the home and connections to the world beyond [2]. Smart Home systems could simply offer additional convenience in everyday activities adding to the benefits provided by the mechanical and electrical technologies. Ubiquitous computing is a technology shift where technology becomes virtually invisible in our lives. Instead of having a desktop or notebook machine, ubiquitous computing will allow technology to become hidden in our lives, built into the things we use, embedded in our environment.

Photographs are a very effective way of connecting people socially. Especially with the invention of digital photography, photo sharing has allowed people to communicate and share emotion through pictures when in a face-to-face environment. Photos provide social interaction with the people we share them with and can form the basis for social relationships as they serve as memory triggers, which allow emotions and memories associated with the pictures to be conjured up. If these personal emotions and memories can be captured by the user and stored with the associated picture hence allowing a digital representation of our emotions, it could make a worthwhile addition to the photo-sharing environment in a smart home. As existing technologies are not designed to support lightweight spontaneous interactions between users especially if they are using the Internet as the communication medium, we think that this approach will make the photo sharing more engaging and less demanding for the user.

The invention of digital cameras has had a profound affect on the way in which we take photographs and share them with friend and families, hence constructing social relationships on the way. Digital photography has allowed us to capture the entire moment of our lives into one photographic image. Photos allow people to share experience and events even if they were not physically there. Today photos are used for a variety of purposes ranging from sharing, decorating homes, albums, as gifts on mugs and photo frames. All these forms of usage shows how people relate themselves to their pictures and how it represents their social lifestyle. 
Advancing technology continues to support families and their endeavours to use photography as a means to support social interaction. The introduction of digital photography, and its rapid acceptance has created a paradigm shift in the way people record and review images of everyday life. Digital cameras have allowed people to capture, reproduce, and share visual narratives more easily [3]. It has allowed people to instantly review the picture taken with easier duplication and sharing, as well as easier disposal of pictures they don't want. This is both economical and eco-friendly as the cost of throwing away digital images is virtually zero.

The home computer has become a digital archive, taking on storage, retrieval and display duties; however, its location in the home and its aesthetic appearance limit the type of sharing and social interactions that happen. For presentation most people still print out physical photos for display and interaction in the home. People do not often change the photos because of the effort involved. Somehow, the digital photos haven't made the full transition to support existing behaviours of people when sharing the photos. Furthermore, current digital photo frames have not yet addressed these needs in the home. The increase in the number of digital photos people are acquiring makes digital display in the home desirable [3].

To help us design photo-sharing environment for a smart home, we needed to get some idea as to what people like to do with photos and how they relate themselves to it. We therefore carried out a survey to measure the attitude of people towards photo sharing. We looked at how people use photographs and how we can make photo sharing easy and fun. The survey dealt with questions regarding the usage of cameras, the living environment, preference for digital or paper storage, medium for photo sharing, significance of a picture, emotional attachment, intimacy, indexing, usage of internet, reception, storage, and distribution. However one of the main objectives of the survey was to see the influence of digital cameras on photo sharing. The findings of the survey can be found in [9].

\subsection{Aim/Purpose}

Photo sharing provides great opportunities for creating and maintaining social relationships within the home. This is due to the fact that we as human beings are social creatures who find human presence and communications essential for the enjoyment of life and since home is a place where we express and share most of our emotions; photo sharing is one way in which these emotions are expressed when we are with friends and family. As proposed by Miller [4] well-being can be supported by encouraging and enabling effective action, prediction and control, satisfying social interaction, and mindfulness, physical involvement and enjoyment. When we look at the home environment most if not all of the factors proposed my Miller could be addressed by providing a photo-sharing environment to address these issues. A central device to handle the reception, storage and distribution would encourage effective action from users as they will be able to access information without being in sight of the source and hence will have control over the information.

The aspect of satisfying social interaction and physical involvement could be resolved by allowing users to attach emotions to pictures associated with them. This would help not only the users in being more involved with pictures but also help 
pictures to act as storyboards and help in creating social relationships. Miller [4] also suggests that an important way of designing for well-being is to design the space that enables people to "do Well-being" for themselves. When we look at the home environment we see the physical space where people spend most of their time and if we could provide an environment where people could relate themselves to some of the attributes of that space, we think it will be a good addition to the smart home.

Looking at the aspects of entertainment within the home, there are several consumer electronic devices that provide multiple interaction mediums to communicate with each other. They include devices such as PC's, Notebooks, PDA's, TV's, Projectors, DVD, and Cameras etc. These multiple interaction mediums form the basis of our space in the home, which we interact with on regular basis and if we bring photo sharing into this space then we need to use these devices. To facilitate a better interaction, we propose a convergence of these technologies and interaction mediums, by providing an In-Home Communication Infrastructure, which can interact with all these devices and provide the comfort and convenience that the user expects.

Although there are digital photo frames which allow users to display images in digital format through the home, what they still lack is in providing ease of use as the user will not always want to use it with friend and families for photo sharing and would prefer a display medium of some other kind like the TV or projector system. If we provide a ubiquitous environment for the user, we cannot simply use one aspect of technology in digital frames, we need to incorporate all the aspects, which the user can associate them with. The home environment should support the same level of rich social interactions and experiences that are associated with traditionally printed photos.

\subsection{Methodology}

The project follows a human-centered design approach in the sense that we carried out a survey to measure the attitude of people towards incorporating digital photo sharing within the home environment. The nature of the home, and the character of everyday home life is undergoing constant change in definition and as such is required to become responsive to the changing needs of people throughout their lifetime. Since our emotions relating to an event or object change over a period of time, a photo-sharing environment, which could capture this change, will allow the user to be constantly involved with photo sharing and will make the technology more fun and enjoyable. Using digital photos for display in the home allows for rich interaction possibilities such as ease of selecting and displaying, annotation and sharing [3]. Although the Cherish framework [3] looks at the social aspect of digital photo frames within the home, it comes short on emotional aspect of pictures and how we can capture emotions of the user in relation to the picture and preserve it digitally.

To help us design photo-sharing software, we needed to get some idea as to what people like to do with photos and how they relate themselves to them. We carried out a survey to measure the attitude of people towards photo sharing. We looked at how people use photographs and how we can make photo sharing easy and fun. The 
survey dealt with questions regarding the usage of cameras, the living environment, preference for digital or paper storage, medium for photo sharing, significance of a picture, emotional attachment, intimacy, indexing, usage of internet, reception, storage, distribution of pictures and how we could integrate the system in an ubiquitous environment. Our research revealed two main opportunities for a photosharing environment in a smart home which would play an important role in the lives of the people living in those homes: -

1. Having a photo-sharing environment to support various devices and media types.

2. To attach and preserve emotions digitally in association with the pictures.

We wanted to know how we could use emotions associated with a particular picture to annotate it for storage and how this annotation could be used for search and retrieval of photos from a database. Our research explores the needs, goals, and desires of families and includes concepts based on the needs discovered, and a concept validation that evaluates if our proposed solution meets the needs of the home owners, as well as if our understanding identifies real needs. The survey results will allow us to generate a smart home concept application that addresses the needs we identified in our fieldwork. Our aim was to validate if the needs we observed matched the needs as families/home owners perceived them. We also aim to probe the receptivity of our proposed solution by users.

\section{Photography and social relationships}

Photographs are a good representation of the social status we enjoy in society. They show our social networking with people. Photos allow presentation of oneself as kind of person one would like to be taken as, and allow other people to go along with that pretence gracefully (Goffman [7]). The photo sharing environment should allow people to feel connected to other people by the way emotions may be expressed in response to their pictures. The social interaction within the home can be increased by providing a photo-sharing environment where the user can attach emotions as well as capture the audio conversation they have when they share pictures with friends and families. This could allow the picture to act as a storyboard for people to see/read/hear who were not present at a particular moment.

Even though there are plenty of photo sharing services available like e-mail, Flickr [16], CONFOTO [15] etc, they are primarily web-based services and are more suitable for people who would take large volumes of pictures of anything and everything. It is also not something people associate with their home, as when you are at home its more about possession of items and things you can physically relate to. This is however dependent on an individual's idea of the home and could vary from person to person. People are less attached to something on the web as compared to physical artefacts that they can see in their home. However as Wise [18] puts it, space (home in this case) can be marked and shaped through physical objects. When it comes to photographs Wise has put forward the idea that photographs glow with 
memories of experience, of history, of family/friends etc, and what creates this glow is the articulation of subject to object, caught up in a mutual becoming home. People are very aware of how their surroundings are viewed by other people and try to express themselves through various objects and possessions. The home environment brings a more personal feel to objects (e.g. a photo frame) for someone who spends most of their time at home. However this could be different for someone whose time is spent in an environment which is not their home but the individual has tried to make space resemble a more homely and friendlier environment (e.g. by putting up photos, posters, objects of interest etc). Wise has forwarded the concept of home as a territory or space to which an individual can add identity through expressions of objects.

A digital photo sharing environment for the smart home will provide friends and families the opportunity to exchange photos as gifts with personal feelings hence supporting the construction of family identity and other social constructs. It could make family members feel better about themselves and the roles they play, and potentially increase the emotional connection between family members.

It could also form part of a communicative technology that might be of use in smart homes. Our research has revealed the following opportunities for this smart home technology to play a role in transforming the lives of the people:

1. By having a photo-sharing environment to support interaction with various devices and media types.

2. To attach and preserve emotions digitally in reference to the picture and allowing the user to capture spontaneous interaction.

3. An evaluation how the use of different data can be used to signal emotions with photographs.

4. Increasing social interactions between people by allowing people who were not present at a particular event to enjoy the experience.

At one time or another we all experience strong feelings that accompany positive or negative emotions. We usually have no problem identifying the emotion that we are experiencing at a given time. Human Beings feel a range of emotions when they view photos. To name a few emotion types of Joy, Distress, Happiness, Sadness, Love, Hate, Envy, Jealousy, Hope, Fear, Satisfaction, Shame, Disappointment etc could be a reasonable addition to pictures. One of the options for users could be providing them with a range of known emotions types and allowing them to select the appropriate emotion to attach to the picture. Event types like seasons, holidays, birthdays, etc could also be used to save pictures.

Another options for users could be saving pictures by relationships with individuals in the picture hence allowing for another retrieval method. People don't want to go through a search structure to get to their photos which can be time consuming and tedious. They want to get to their pictures as quickly as possible. Our emotions can be put into groups, which contain various emotion types experienced, by people on a day-to-day basis. These groups can be categorised as Well-Being, Fortunes-of-Others, Prospect-Based, Confirmation, Attribution, Attraction, WellBeing/Attribution, and Attraction/Attribution. The category labels and emotion types corresponding to each group have been summarised in [14]. We need to map this 
grouping of emotions with the mental model of the user so that it's easy for them to recognise the particular emotion they are feeling when looking at a picture and affectively attach it. Our picture could sometimes invoke multiple emotions at the same time e.g. feeling of fear and excitement (Could be at various levels) at the same time when doing some extreme sport.

Our photos allow us to reflect back on the past, cater for present and to some extent plan for the future regarding our family/friends, hence looking forward to creating new memories to be shared. Storytelling and reminiscing using photographs are one way of keeping and sharing memories and building family legacy [12]. The respondents from our earlier survey [9] favoured our concept of a photo-sharing environment in a smart home, which could provide reminders about the change in emotions in association with pictures over a period of time as well as changes in our daily routines.

The invention of wireless digital camera's and wireless photo frames will allow for the environment to become ubiquitous as then the user can manage the images through the central device. This will allow the user to, for example display images, which are stored in his camera directly to the photo frame or on any other display medium.

\section{Photo sharing In Smart Homes}

Home is a place associated very closely with human beings. It's a place where we spend most of our time and interact closely with our friends and family. Previous research has focused on how smart homes can improve human experiences, however those researches have focussed mainly on controlling devices and less on allowing people more control over their lives. One of the things people value the most in their homes is their collection of photos as it represents their social contacts and life events. As revealed from our earlier survey [9], photos bring out emotions in people and they would like digital technology to represent personal emotions on pictures and get emotional satisfaction from the thing they value most.

Digital data coming into the home today includes music in CD-format, video in DVD, digital photos, and there is already digital radio and TV-channels. Multimedia Entertainment is slowly becoming more and more readily available which is allowing the users to experience a more augmented experience. In order to make it as easy as possible for the occupants of the smart house to interact with the various smart appliances, the medium of communication must be as natural as possible [9]. Sloane et al [5] summarize the range of information used in the home and outlines the movement of information and the actors involved in its use. It also shows the diversity of information usage in the home. Our survey revealed that digital technology has had a great impact on digital photography and has allowed more people to become involved with photo sharing Fig 1. With the advent of the Internet and with it becoming a part of more and more people's lives, sharing photos with friends and families has become easier. 


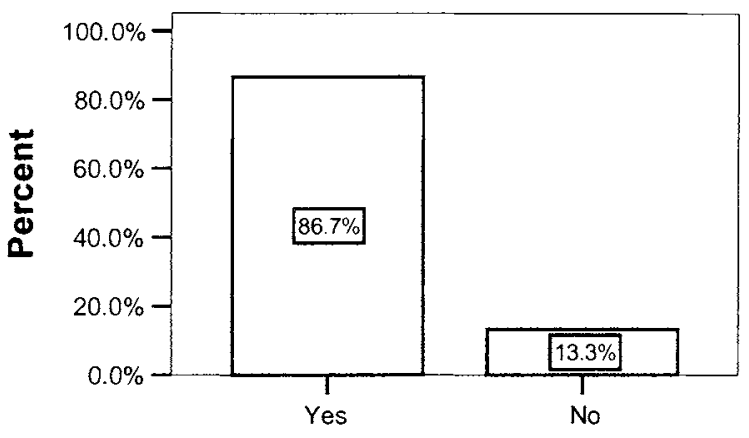

Fig. 1. Digital Technology has made Photo sharing easier.

We need to understand the roles of the smart home as well as what part presentation of digital photos have to play in our surroundings. In the future where the communication between various devices will be ubiquitous, it will become easier for the user to interact with the data on devices, which are not in their line of sight. When it comes to photo sharing our central device will allow the user to receive, store, distribute and view the pictures without having to interact with the actual device on which the pictures were originally stored. We hope that our research will help us in providing a framework for a smart home environment where photo sharing and emotional attachment to the pictures can be represented in a digital format.

The survey [9] helped in finding out the expectations, needs and desires of the users and will help us in creating a sense of desirability for the application. Our photo-sharing environment will allow them to achieve the sense of who they are and how they would like to be represented. By providing the users with a compelling and entertaining environment to share pictures in, we could create a sense of comfort and familiarity with the user that we hope fosters increased usage of the application and further enjoyment of their pictures and memories. This could be achieved by providing a high degree of on screen activity for the users to engage themselves in.

When we bring together the technologies involved in smart home of the future and a photo-sharing environment, there should be clear guidelines as to what this kind of technology should provide the users with. Friedewald et al [7] points out what we need to keep in mind when developing smart homes: -

1. It helps its inhabitants live a healthy, happy and safe life.

2. It performs tasks automatically to relieve the stress of managing the house.

3. It integrates home, work, learning and leisure activities.

4. It does not annoy people with the technological details of how it actually works. 


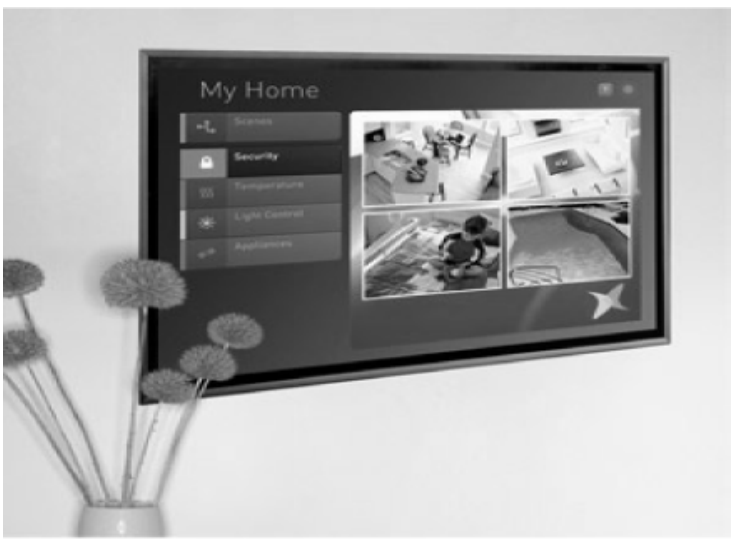

Fig. 2. Home automation solutions provided by ConvergeX

The above points to some extent have been realised by researchers of technologies who are now working with many developers to achieve these in the home environment. An example of this is the ConvergeX [20] home automation platform in the UK (Figure 2), which provides home automation services for new properties being developed as well as the old properties where owners want their technology platforms to adapt to their specific requirements. However Friedewald's suggestions are mainly technical and cover the technological aspects of the technology. They fall short on the emotional domain when it comes to building technology in the smart homes, which could assist with our emotional attachment to objects/space. Our research extends the above technical details to incorporate the following features:

1. To support the affective well being of the inhabitants, and

2. To support building/maintaining of interpersonal relationships.

If the photo sharing system could absorb some of these guidelines for providing the user with a ubiquitous environment to share photos with friends/family and allow them to attach emotions to pictures digitally, it should provide for a fun and enjoyable experience. The key for a successful photo-sharing environment lies with designing a product that is both entertaining and emotionally engaging. This could be achieved by making the picture a central feature of the application with which the user can interact with hence allowing the user to experience the sharing environment and the memories that the pictures often facilitate. 


\section{Emotions and Digital Photo Sharing in a Smart Home}

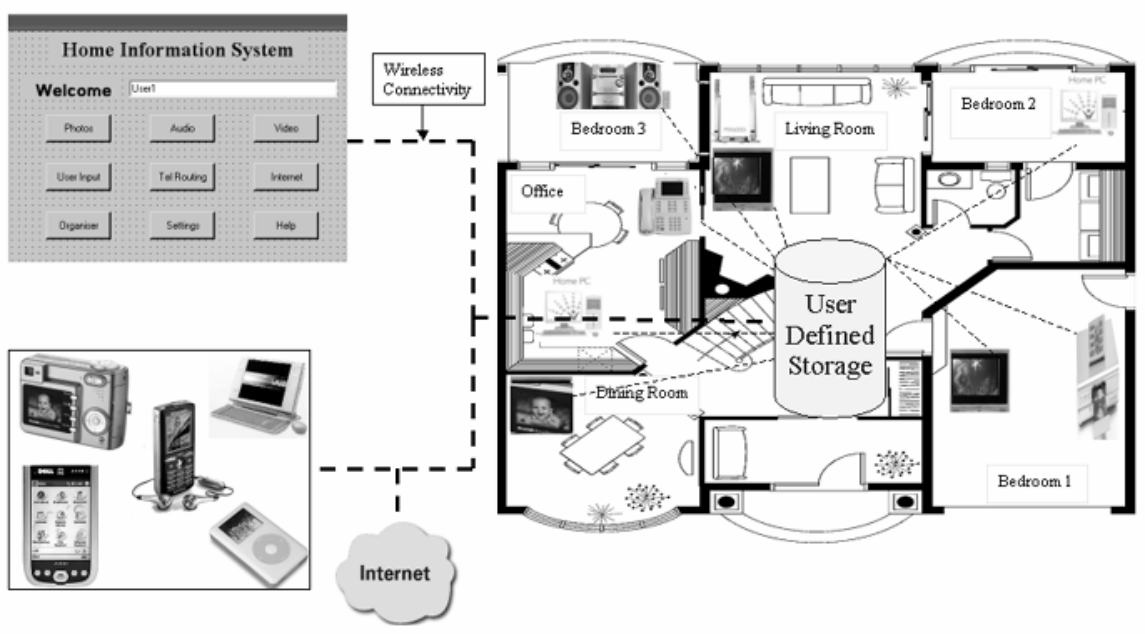

Fig. 3. Digital Photo sharing in a networked smart home.

The scenario in Fig 3 will require interaction with various communication/networking mediums. What the HIS will provide is a simpler way to interact with these devices for the home user i.e. if the user wants to transfer data from one device to another which are located in different location in the home without actually having to physically interact with the devices, the HIS will allow for this facility. For example, if the PC is located in the bedroom and is Wi-Fi enabled, the user can use the HIS (in the living room) to communicate with it and transfer data from the PC to the central storage and then view it on the TV as a slide show. The storage requirements of data could vary from one person to another and the scenario needs to be incorporated in the design of the HIS with options for future expansion regarding storage requirements.

The most immediately apparent difference between looking at static images on a web page and sitting down with your friend and a stack of photos is the absence of voice. Speaking while sharing photos is not only natural, but is also socially expected [11]. Our survey [9] revealed that adding text and audio to the pictures will help in elaborating the memories associated with photos. It will help attaching emotions to the pictures. The users could add text by using a standard keypad, writing by hand to give it a more personal feel, or by making use of the voice-to-text technology. This could be used to annotate picture by the user and could be used for search and retrieval of pictures from the database using the comments as a search criteria.

The scenario in Fig 4 shows how a user might receive photos for storage from another device in the house. This could involve a user trying to download pictures from a digital camera, which might be in the one of the rooms in the house to the central server using $\mathrm{Wi}-\mathrm{Fi}$ as the communicating medium. The user will have option of creating a new folder or saving in an existing folder on the server database. Once this is completed, the user can decide if they want to download the whole collection 
from the camera at one go or view and download each picture individually. This will give them the option of only storing the pictures they want and rejecting other pictures. It will also allow the users to name the pictures while they are being downloaded individually rather than the pictures being saved with some random number with a ".JPEG" extension. This was evident from our survey results in [9] where respondents were very positive about the scenario of providing them the option of downloading individual pictures and naming them at the same time from the source storage. Although there are other options apart from the name, which could be added to the picture while its being downloaded, e.g. place, event, relationship etc, we thought we would provide these options to the user when they view these pictures or want to make changes to them, or attach emotions. However if the users suggest that they would like to attach some more information to the pictures while they are being downloaded, the interface could be changed to accommodate that requirement.

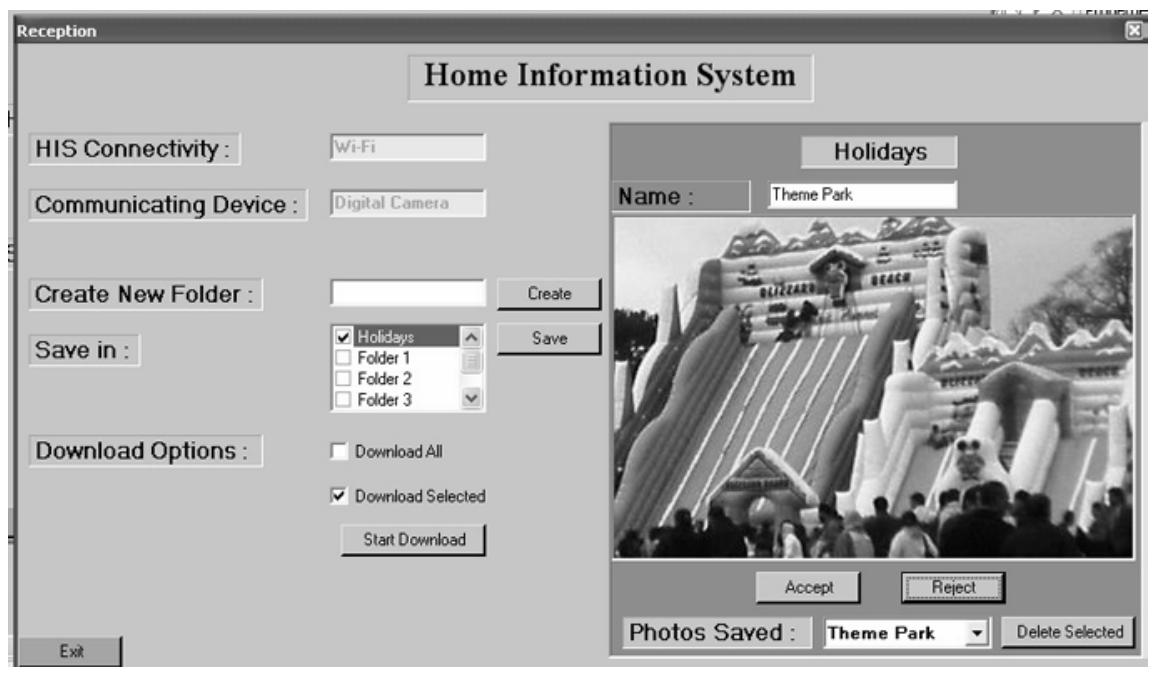

Fig. 4. Receiving photos from a device for storage on the central server

Viewing of pictures and adding emotions through different options available to users will allow them to feel more connected with the pictures and the task they aim to achieve i.e. of photo sharing in a home environment. The user will be able to select the directory in which the pictures are stored to view them and have access to various options like maximising the photo, deleting, editing, adding emotions, emailing and saving the changes. The picture will also represent the emotions, audio, or the text that the user has attached to it, although the best way to represent these is still undergoing changes and will form the basis for our next survey when the users test it. Fig 5 shows the "View Photo" screenshot where the user can select the source where the pictures are saved, select the folder they want to view, and read the text associated with that picture. 


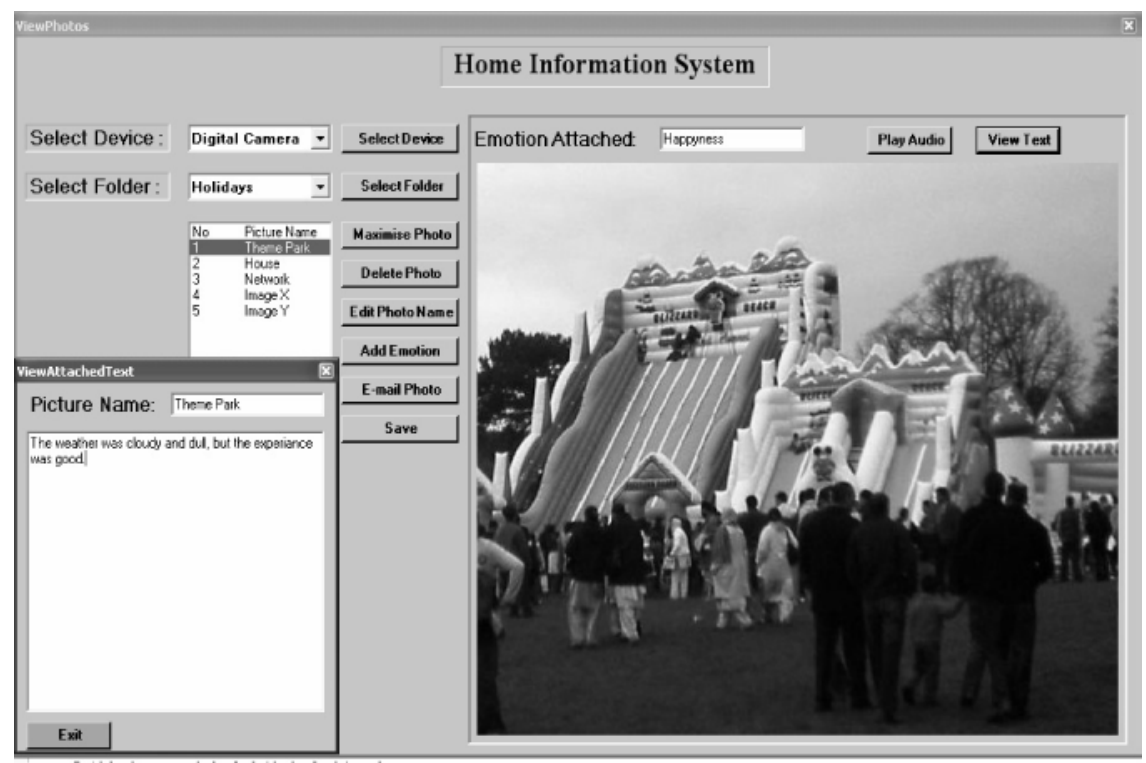

Fig. 5. Viewing the photos stored on the central server.

Recognising that a picture is a memory also implies that pictures can be a nexus of communal bonds. Because of this, it is critical that the application address the basic human need to connect with one another in a compelling way [10]. Digital photo sharing in a smart home will allow family members and friends to forge a bond with each other that reinforces and leverages the unique connection between memories and important moments in their lives. It will provide users with a central point from which memories can be relived and shared with others as well as record their emotional attachment with the picture digitally.

Attaching emotions or creating a storyboard with a picture depends on the type of picture the user/viewers are looking at. The picture could be to do with a place visited, an event, family photo, moments in life, work, pleasure etc. Although there are some pictures, which will have the same emotional response from people, some pictures can generate different types of emotions in different people. To cater for this variation we are trying to give the user the option of attaching emotions based not only on different emotional categories, but also based on relationship types and the other attributes mentioned earlier that could be associated with the picture to elicit emotions. The user will also have the option of attaching an audio story with the picture, which will help build social relationships or strengthen an existing relationship. Audio will also help people describe an event in a way, which might not be available through the standard emotional categories. Fig 6 tries to demonstrate some of the options available to users for attaching emotions to photos. 


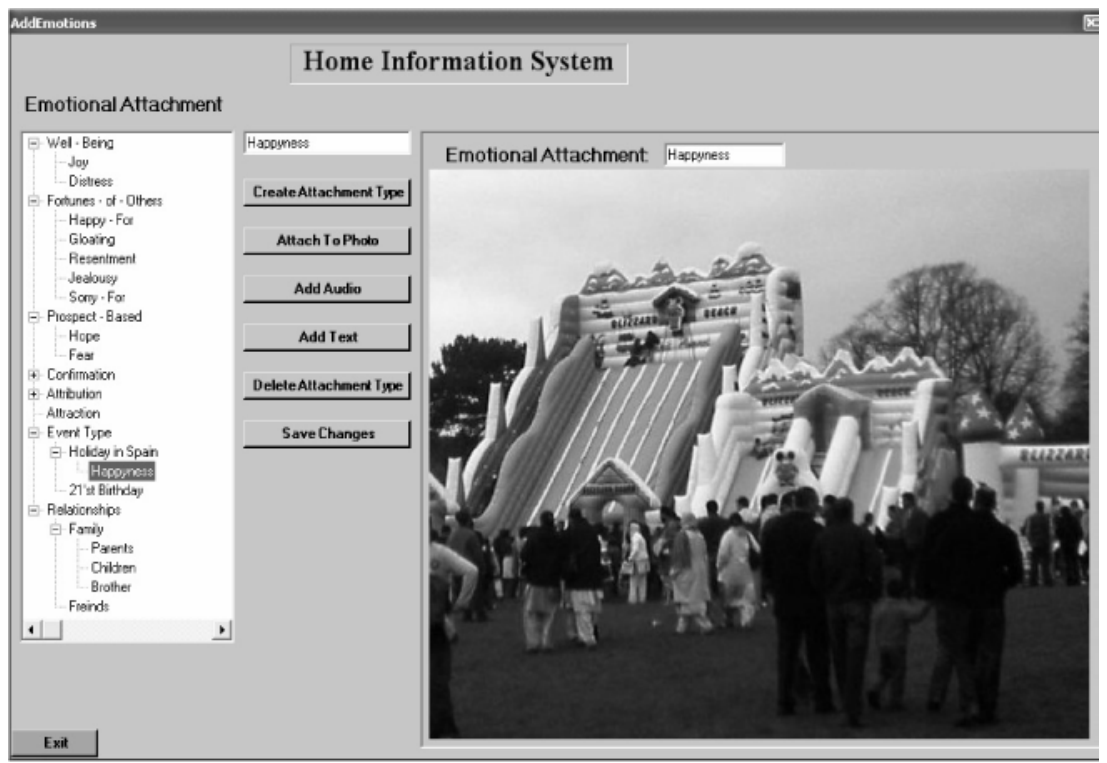

Fig. 6. Attaching emotions to pictures.

We now have digital cameras, which are equipped with some form of wireless connection. This will allow us to send the picture directly form the camera to the central server or view them on the handheld device. It will also allow photos to be displayed on display mediums directly from the camera as long as they are connected by a network connection. The figure below illustrates how such distribution of photos within the networked home might occur where the user might select a source device, which holds the pictures, and then view those pictures on a viewable platform as a slide show or send them to be saved on another device.
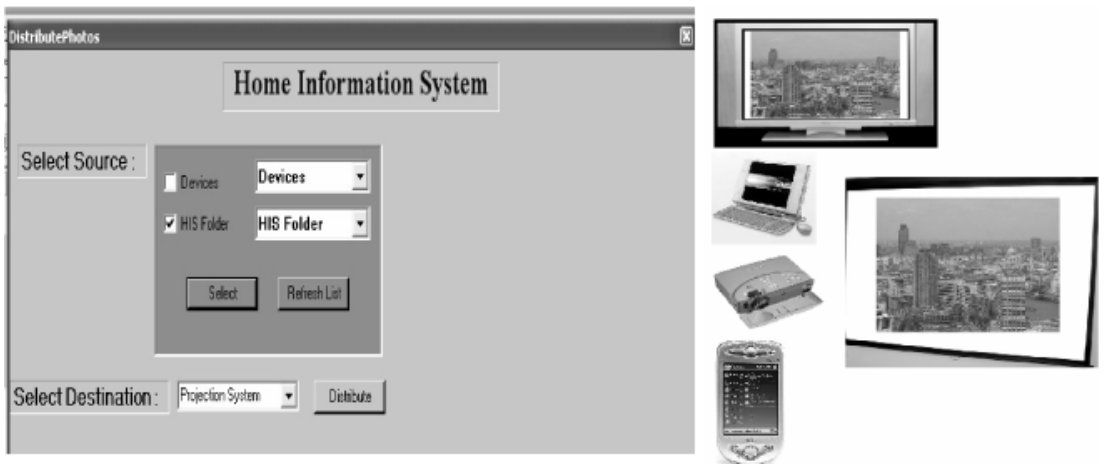

Fig. 7. Distributing images to other platforms for viewing. 
Emotions are mental states that arise spontaneously, rather than through conscious effort. Emotions are physical expressions, often involuntary, related to feelings, perceptions or beliefs about elements, objects or relations between them, in reality or in the imagination [5]. One problem with existing technologies is that they often require too much time and effort and are not designed to support lightweight, spontaneous interactions [6], which might occur while sharing photos. By making photo sharing less demanding and more engaging for the user, we can provide better support for people to people interactions. Emotions are very volatile and can be easily dispersed by a badly designed user interface. Emotions are also highly personal and subjective, which makes it difficult to test in a controlled fashion as they vary widely from one individual to another.

Emotional bonds tie family members together. These emotional bonds are reinforced by "family activities", such as Sunday dinner or vacations together [17]. Our photo-sharing environment within a smart home could enable easy creation and retrieval of memories (in the form of Pictures). This could ultimately reinforce the emotional bonds among family members. Kim et al [3] suggests that women are the primary organisers of photos for storage and display in the home and males share in taking photos with women. Once the photos were taken, it is mostly the women who spent more time preparing and sharing photos with friends and families. Their suggestion is valid as in most societies women are seen as the homemaker and may want to present her lifestyle through pictures.

\section{Conclusion}

The home of the future will not just be about devices in the home or embedded in the fabric. It will bring together other aspects of technology like wearable computing, smart clothing, multimedia systems, positioning systems, and entertainment computing etc. Sloane [13] also points out problem with large scale of legacy information, which could be in different formats. The legacy data needs to be brought together into the standard format. This digitisation will require time and effort, which may ultimately determine the initial take-up of HIS in the market.

The photo-sharing environment would provide a long-term engagement and satisfaction for the user, as people get bored very quickly of performing the same task over and over again. This could be achieved by involving the user with tasks and activities which are ever changing, and when we look at the relationship between emotions and digital photos it becomes clear that since our emotions change over a period of time, it could be just the factor which will allow the user to be involved with the environment for longer. Miller [4] suggests that products, which give, clear guidance about how they can be used, and allow a sense of control and predictability, will support satisfying interaction.

The aim of this project is to come up with design specifications for a home information system, which allows for the integration of digital media technologies into a seamless, cohesive environment. The research has gone according to the goals set at the beginning. The respondents in the survey have reinforced our initial thought that people want to attach emotions to pictures. We wanted to know about what people want from photo sharing, what they enjoy, how specific design 
attributes will make them feel, what will delight them and how through this design process their experience might be enhanced. We are now into the development phase of the system, in which we will provide a relaxed atmosphere to view the photos in. We aim to provide the users with photo sharing software where the users can attach emotions to the digital pictures, use personal annotations to store and retrieve pictures from a database, and view then on different viewable platforms.

\section{References}

1. M. Friedewald, O. Da Costa, Y. Punie, P. Alahuhta, S. Heinonen, Perspective of ambient intelligence in the home environment. Telematics and Informatics, 2005.

2. J Barlow, \& T. Venables, Smart Home, Dumb Suppliers? The Future of Smart Homes Markets, in R. Harper (ed ) Inside the Smart Home, Springer, ISBN 1-85233-688-9, 2003.

3. Jeong Kim, John Zimmerman, Cherish: Smart Digital Photo Frames. Design And Emotion 2006 The fifth conference on Design and Emotion, Gothenburg, Sweden on September 27-29, 2006.

4. Hugh Miller, Mirja Kalviainen, Design for Wellbeing. Design And Emotion 2006 The fifth conference on Design and Emotion, Gothenburg, Sweden on September 27-29, 2006.

5. Friedemann Mattern, Eth Zurich, Wireless Future: Ubiquitous Computing, http://www.vs.inf.ethz.ch/publ/papers/mattern2004_electronica.pdf, (2004).

6. Oliver Liechti, Tadao Ichikawa, A Digital Photography Framework Supporting Social Interaction and Affective Awareness. HUC1999, Karlsruhe, Germany, (1999).

7. Goffman, E. The Presentation of Self in Everyday Life. New York: Doubleday Anchor, (1959).

8. http://www.convergex.co.uk/

9. Sondhi G, Sloane A, Emotions And Digital Photo Sharing In A Networked Smart Home, D\&E, Gothenburg, Sweden, (2006).

10. Wood T, Next Generation Software User Experience at Eastman Kodak Company, D\&E, Gothenburg, Sweden, (2006).

11. Chalfen, R. "Snapshot Versions of Life", Bowling Green State University Press, 1987

12. McAdams D.P. The Stories We Live By. Personal Myths and the Making of the Self. Guildford Press, (1993).

13. Sloane A, Harris A and Huang W (2000b) "Home information systems: the storage problem" in Sloane A and van Rijn F (Eds.) "Home Informatics and Telematics: Information, Technology and Society", Kluwer Academic publishers, Boston MA. ISBN 0-7923-7867-9

14. http://condor.depaul.edu/ elliott/emotion-table.html

15. http://www.confoto.org/home

16. http://www.flickr.com/ 
17. Darrah, C. N., English-Lueck, J. \& Freeman, J. Families at work: An ethnography of dual career families, Report for the Sloane Foundation (Grant Number 98-6-21), (2001).

18. J. Macgregor Wise, HOME: TERRITORY AND IDENTITY, Cultural Studies, Volume 14, Number 2 / April 1, 2000, pages 295-310. 